\title{
m6A Methylation Mediates LHPP Acetylation as a Tumour Aerobic Glycolysis Suppressor to Improve the Prognosis of Gastric Cancer
}

Jian-Xian Lin

Fujian Medical University Union Hospital

Ning-Zi Lian

Fujian Medical University Union Hospital

You-Xin Gao

Fujian Medical University Union Hospital

Qing-Zhu Qiu

Fujian Medical University Union Hospital

Hua-Gen Wang

Fujian Medical University Union Hospital

Qiao-Ling Zheng

Fujian Medical University Union Hospital

Ying-Hong Yang

Fujian Medical University Union Hospital

\section{Yu-Bin Ma}

Qinghai University Affiliated Hospital

Chao-Hui Zheng

Fujian Medical University Union Hospital

Ping Li

Fujian Medical University Union Hospital

Jian-Wei Xie

Fujian Medical University Union Hospital

Jun Lu

Fujian Medical University Union Hospital

Qi-Yue Chen

Fujian Medical University Union Hospital

\section{Long-Long Cao}

Fujian Medical University Union Hospital

Mi Lin

Fujian Medical University Union Hospital

Jia-Bin Wang 
Fujian Medical University Union Hospital

Chang-Ming Huang ( $\sim$ hcmlr2002@163.com )

Fujian Medical University Union Hospital https://orcid.org/0000-0002-0019-885X

\section{Research Article}

Keywords: Methylation Mediates, Tumour Aerobic Glycolysis, Gastric Cancer

Posted Date: July 15th, 2021

DOl: https://doi.org/10.21203/rs.3.rs-680175/v1

License: (c) (1) This work is licensed under a Creative Commons Attribution 4.0 International License. Read Full License 


\section{Abstract}

\section{Background}

LHPP, a histidine phosphatase, has been implicated in tumor progression. However, its role, underlying mechanisms, and prognostic significance in human gastric cancer (GC) are elusive.

\section{Methods}

We obtained GC tissues and corresponding normal tissues from 8 patients and identified LHPP as a downregulated gene via RNA-seq. qRT-PCR and western blotting were applied to examine LHPP levels in normal and GC tissues. The prognostic value of LHPP was elucidated using tissue microarray and IHC analyses in two independent GC cohorts. The functional roles and mechanistic insights of LHPP in GC growth and metastasis were evaluated in vitro and in vivo.

Results

The results showed that LHPP expression was significantly decreased in GC tissues at both the mRNA and protein level. Multivariate Cox regression analysis revealed that LHPP was an independent prognostic factor and effective predictor in patients with GC. The low expression of LHPP was significantly related to the poor prognosis and chemotherapy sensitivity of gastric cancer patients. Moreover, elevated LHPP expression effectively suppressed GC growth and metastasis in vitro and in vivo. Mechanistically, the m6A modification of LHPP mRNA by METTL14 represses its expression; LHPP inhibits the phosphorylation of GSK3b through acetylation, and mediates HIF1A to inhibit glycolysis, proliferation, invasion and metastasis of gastric cancer cells.

\section{Conclusion}

LHPP is regulated by m6A methylation and regulates the metabolism of GC by changing the acetylation level. Thus, LHPP is a potential predictive biomarker and therapeutic target for GC.

\section{Background}

Gastric cancer (GC) is the third most common tumour-related disease worldwide [1, 2]. Cancer cells need to effectively coordinate glycolysis and glutamate decomposition to meet their bioenergy and biosynthesis requirements for proliferation and survival $[3,4]$. However, the metabolic adaptations of GC and the mechanisms of tumour proliferation, invasion, and metastasis have not been fully elucidated, and there is a lack of specific and efficient intervention methods in clinical practice $[5,6]$. Therefore, identifying molecular markers and their mechanism of action which inhibits the metabolic adaptation, invasion, and metastasis of GC can provide new targeted therapies which have important theoretical and clinical significance. 
Phospholysine phosphohistidine inorganic pyrophosphate phosphatase (LHPP) is a histidine phosphatase, whose role is opposite to that of histidine kinase, which can remove protein and histamine acid-linked phosphate groups. The human LHPP gene (NM_022126) is located on chromosome 10 (10q26.13). It is an acidic protein containing 270 amino acids and is a nontransmembrane protein which is mainly located in the cytoplasm and is expressed in most tissues [8-10]. Previous studies have found that $\angle H P P$ is a genetic marker of alcohol dependence and severe depression and is related to mitochondrial dysfunction and chronic oxidative stress [10,11]. Two genome-wide association studies have found that $L H P P$ is a susceptibility gene for primary open-angle glaucoma, oropharyngeal cancer, and acute lymph node cell leukaemia $[12,13]$. A study by Hindupur et al. reported that overexpression of LHPP can inhibit liver tumour formation, maintain liver function, and is related to patient prognosis [14]. However, whether and how LHPP regulates metabolic adaptation in GC remains unclear.

By investigating the upstream regulatory mechanisms of LHPP, we found that its mRNA level may be related to N6-methyladenosine $(\mathrm{m} 6 \mathrm{~A})$ methylation modification. m6A methylation is the most prevalent internal chemical modification of eukaryotic mRNAs [15]. Previous studies have reported that the effects of m6A modification include regulating mRNA stability, splicing, and translation [16]. Methyltransferaselike 14 (METTL14) is a catalytic enzyme which promotes m6A modification of mRNA [17]. However, the mechanism by which METTL14 mediates the m6A modification of LHPP to affect GC progression has not yet been reported.

Bioinformatics analyses of public databases revealed that LHPP may affect tumour glycolysis through acetylation. The prominent feature of tumour cell metabolic adaptation is the Warburg effect, that is, regardless of the oxygen state, many cancer cells rely on high glucose uptake rates and convert most of the glucose into lactic acid through glycolysis rather than catabolising glucose through oxidative phosphorylation [18]. The Warburg effect may be caused by the hypoxic microenvironment of the tumour, abnormal signalling pathways, abnormal activation of oncogenes, glucose transporters, or the overexpression of enzymes in the glycolytic pathway [19].

In this study, we investigated the clinical significance and regulatory mechanism of LHPP in GC.

\section{Materials And Methods}

\section{Patients and Gastric Tissue Sample}

The study was primarily based on 2 independent patient cohorts. Cohort 1 included 349 gastric cancer tissues collected from January 2010 to April 2014 at Fujian Medical University Union Hospital. Gastric tissue specimens included tumor tissues of the stomach and adjacent non-tumor tissues. Cohort 2 included 93 gastric cancer tissues collected from January 2010 to April 2014 at Qinghai University Hospital for external validation. The inclusion criteria were as follows: (a) histological identification of gastric cancer; (b) availability of follow-up data and clinicopathological characteristics; (c) TNM staging of gastric cancer tumors according to the 2010 International Union Against Cancer (UICC) guidelines. The 
exclusion criteria were as follows: (1) patients with no formalin-fixed paraffin-embedded (FFPE) tumor sample (CT and IM) from initial diagnosis; (2) patients who received chemotherapy or radiotherapy before surgery. All participating patients with advanced GC routinely received fluorine-based chemotherapy (Table 1).

\section{Construction of Tissue microarray (TMA)}

From January 2010 to April 2014, a total of 123 gastric cancer tissue samples were selected. Briefly, the pathologist examined all gastric cancer tissues, and marked the paraffin blocks based on the tumor position of the HE stained section and immunohistochemical slides, and selected more areas of the tumor tissue without the representation of necrosis and hemorrhagic material Area to prepare tissue chips for experiments. Mix paraffin wax and an equal amount of beeswax to make 2 blank wax blocks. Create a puncture hole with a diameter of $1 \mathrm{~mm}$ in blank paraffin to separate the two holes, and perform 80 punches. For each sample, a $1.5 \mathrm{~mm}$ core was punched from the donor block using a tissue microarray instrument. Use a tissue analyzer to sample the tumor-marked wax block, put the sampled tissue into the corresponding channel of the blank wax block, and transfer the determined array position to the recipient paraffin block. Several serial sections ( $4 \mu \mathrm{m}$ thick) were cut from all TMAs, and one section of each TMA was stained with hematoxylin-eosin to ensure that the TMA was constructed correctly. The intratumor dot was derived from the center of the tumor, while the peritumor dot was punched out from the area $\geq 2 \mathrm{~cm}$ from the tumor margin. The prepared TMA slides are used for immunohistochemistry (IHC).

\section{Follow-up}

All patients were systematically followed up by trained doctors who abided by the institutional follow-up protocol; options for follow-up included outpatient services, letters, telephone, mail or visits. Follow-up was conducted every 3-6 months for the first 2 years, every 6-12 months for the 3-5 years, and annually thereafter. Survival time was defined as the time from the date of surgery until the date of last follow-up or death. All 565 patients involved in the IHC analysis completed the follow-up.

\section{Immunohistochemistry (IHC)}

The serial sections of the FFPE sample were $4 \mu \mathrm{m}$ and mounted on a glass slide for IHC analysis. The sections were deparaffinized with xylene and rehydrated with alcohol. We blocked the endogenous peroxidase by immersing the slices in a $3 \% \mathrm{H} 2 \mathrm{O} 2$ aqueous solution for 10 minutes and microwaved them in $0.01 \mathrm{~mol} / \mathrm{L}$ sodium citrate buffer $(\mathrm{pH} 6.0)$ for 10 minutes for antigen retrieval. The slides were then washed in phosphate-buffered saline (PBS) and then incubated with 10\% normal goat serum (Zhongshan Biotechnology Co., Ltd., China) to eliminate non-specific reactions. Subsequently, the primary antibody was incubated with the antibody overnight at $4^{\circ} \mathrm{C}$. The treatment of the negative control is the same, but the primary antibody is omitted. After rinsing 3 times with PBS, dilute the slide and secondary antibody for 30 minutes at room temperature, and develop with diaminobenzidine (DAB) solution. Finally, the slides were counter-stained with hematoxylin, dehydrated, and fixed with a cover glass and neutral resin. 
We performed LHPP (NBP1-83273, Novus, 1:300), METTL14 (ab220030, abcam, 1:1000), HIF-1a (ab243861, abcam, 1:500), GSK-3ß (phospho S9) (ab75814, abcam, 1:200) immunohistochemical staining on the tumor tissue of gastric cancer patients. The staining intensity and average percentage of positive cells in 5 randomly selected regions were evaluated to represent the protein expression level. The scoring criteria are as follows: staining intensity is divided into 0 (negative staining), 1 (weak staining, light yellow), 2 (medium staining, yellow-brown) or 3 (strong staining, brown), and positive staining of tumor cells The proportion is divided into 0 ( $\leq 5 \%$ positive cells), 1 (6\%-25\% positive cells), 2 ( $26 \%-50 \%$ positive cells) or 3 ( $\geq 51 \%$ positive cells) cells). The final expression is calculated by multiplying the staining intensity score by the proportional staining score (total 0 to 9 ). Patients with final scores of 0,1 , 2, and 3 were classified as low expression group, and patients with scores 4, 6, and 9 were classified as high expression group.

The IHC results were evaluated by two independent gastroenterology pathologists who were blinded to the clinical data prognosis of the patients. Approximately $90 \%$ of the scoring results are the same. When the scores of the two independent pathologists diverged, another pathologist checked the results again and chose one of the scores of the first two doctors, or the three pathologists discussed the decision together.

\section{Gene set enrichment analysis}

Gene set enrichment analysis (GSEA) performed by the Molecular Signature Database (MSigDB) was used to identify the pathways and functions that were significantly enriched in LHPP low tumor samples. If a gene set had a positive enrichment score, the majority of its members had higher expression accompanied with higher risk score, and the set was termed 'enriched'.

\section{Statistical analysis}

All data were processed using SPSS 25.0 (SPSS Inc. Chicago, IL) and R software (version 4.0.0). The Kaplan-Meier method was used to estimate median survival. We defined the survival time of patients who were lost to follow-up as the time from surgery to the last follow-up time, and the survival time of patients who were still alive in the end was defined as the time from surgery to the database deadline. A two-tailed $P$ values $<0.05$ were considered significant differences.

\section{RNA-sequencing analysis}

Total RNA extraction was performed with TRIzol Reagent (Invitrogen, Carlsbad, CA, USA). RNAsequencing analysis was performed at KangChen Bio-tech Inc. (Shanghai, China).

\section{Establishment of cell lines}

Overexpression and knockdown lentiviruses for LHPP (NM_022126.4) as well as control lentivirus were purchased from GeneChem Corporation (Shanghai, China). Transfection was performed according to the manufacturer's instructions. Puromycin ( $2 \mu \mathrm{g} / \mathrm{ml}$, Sigma) was used to select stable clones for at least 1 
week. At the indicated time points, the cells were harvested for mRNA and protein analysis as well as for other assays.

\section{RNA immunoprecipitation (RIP)}

LHPP m6A immunoprecipitation was performed using a Magna MeRIP m6A Kit (17-10499, Merck Millipore, USA) according to the manufacturer's protocol, and the immunoprecipitated RNA extracts were reversetranscribed and analyzed by qRT-PCR.

\section{Human Phospho-kinase Array}

The relative levels of protein phosphorylation were tested using the Human Phospho-Kinase Array Kit (ARY003B, R\&D Systems, Inc. USA \& Canada) according to the manufacturer's protocol. An equal amount of protein (600 mg) was extracted from stable cells (Overexpression LHPP HGC-27 and Control HGC-27) and used to compare the kinase activity with or without LHPP overexpression.

\section{Tumor formation and metastasis assays}

All male BALB/c nude mice (4-5 weeks old) used in our study were purchased from Beijing Vital River Laboratory Animal Technology Co., Ltd. A total of $5 \times 106$ stably transfected MGC-803 cells were subcutaneously injected into the right axillary fossa of nude mice. Tumor volume was measured every 3 days and calculated with the following formula: $V=(L \times W 2) / 2 \mathrm{~cm} 2(V$, tumor volume; $L$, length; $W$, width $)$. The mice were sacrificed at 3-4 weeks after injection, and the tumors were weighed. For the lung or liver metastasis model, $5 \times 106$ stably transfected MGC-803 cells were injected into the tail veins or the spleen of nude mice. Forty-five days later, the mice were sacrificed, and the lungs or the livers were dissected to examine the histopathological metastatic loci. The peritoneal dissemination ability of GC cells was evaluated via intraperitoneal injection. A total of $5 \times 106$ stably transfected MGC-803 cells in $500 \mu$ of PBS were injected into the peritoneal cavity of BALB/c nude mice. Mice were carefully monitored until they were killed at 4 weeks, at which point peritoneal metastases were examined and recorded. All animal experiments were performed according to the Animal Protection Committee of Fujian Medical University (Fuzhou, China) and approved by the Ethics Committee of Fujian Medical University/Laboratory Animal Center (Fuzhou, China).

\section{Western blot assay}

Samples and cells were collected for Western blotting as previously described. Western blot analysis was performed using the following antibodies: LHPP (NBP1-83273, Novus, $0.2 \mathrm{ug} / \mathrm{ml}$ ), METTL14 (ab220030, abcam, 1:1000), HIF-1a (ab243861, abcam, 1:1000), $\beta$-ACTIN (ab8226, abcam, 1:2000), Acetyl Lysine (ab190479, abcam, 1:1000), P300 (ab10485, abcam, 1:5000), TIP60 (ab151432, abcam, 1:1000), GCN5 (ab282176, abcam, 1:1000), PCAF (ab176316, abcam, 1:1000), GLUT1 (ab115730, abcam, 1:5000), cMyc (ab32072, abcam, 1:1000), PKM2 (ab137852, abcam, 1:1000), ALDOLASE (ab252953, abcam, 1:1000), ENOLASE1 (H00002023-M01, Novus, 1:500), GLS1 (H00002744-M01, Novus, 1:500), GSK-3 $\beta$ 
(phospho S9) (ab75814, abcam, 1:5000), GSK-3ß (ab32391, abcam, 1:5000), $\beta$-CATENIN (phospho S37) (ab75777, abcam, 1:500).

Table 1. Relationship Between LHPP Expression and Baseline Characteristics of Patients 


\begin{tabular}{|c|c|c|c|c|c|c|c|}
\hline \multirow{3}{*}{$\begin{array}{l}\text { variables } \\
\text { All patients }\end{array}$} & \multicolumn{4}{|c|}{ Internal Set } & \multicolumn{3}{|c|}{ External Set } \\
\hline & \multicolumn{2}{|c|}{ LHPPlow } & \multirow{2}{*}{$\begin{array}{l}\text { LHPPhigh } \\
5\end{array}$} & \multirow[t]{2}{*}{$P$} & \multicolumn{2}{|c|}{ LHPPlow } & \multirow[t]{2}{*}{ LHPPhigh } \\
\hline & 214 & 135 & & & 53 & 40 & \\
\hline Gender & & & 0.58 & & & & 0.816 \\
\hline Female & 53 & 35 & & & 15 & 12 & \\
\hline Male & 161 & 100 & & & 38 & 28 & \\
\hline Age at surgery(yr) & & & 0.02 & & & & 0.454 \\
\hline$<65$ & 126 & 77 & & & 39 & 33 & \\
\hline$\geq 65$ & 88 & 58 & & & 14 & 7 & \\
\hline BMI & & & 0.45 & & & & 0.555 \\
\hline$<25$ & 186 & 113 & & & 44 & 37 & \\
\hline$\geq 25$ & 28 & 22 & & & 9 & 3 & \\
\hline TNM stage & & & 0.00 & & & & 0.001 \\
\hline I & 24 & 23 & & & 11 & 9 & \\
\hline ॥ & 74 & 43 & & & 19 & 15 & \\
\hline III & 116 & 69 & & & 23 & 16 & \\
\hline Chemotherapy* & & & 0.00 & & & & 0.013 \\
\hline No & 100 & 77 & & & 31 & 16 & \\
\hline Yes & 114 & 58 & & & 22 & 24 & \\
\hline Tumor size (mm) & & & 0.00 & & & & 0.030 \\
\hline$\leq 40$ & 77 & 57 & & & 26 & 25 & \\
\hline$>40$ & 137 & 78 & & & 27 & 15 & \\
\hline Resection type & & & 0.33 & & & & 0.167 \\
\hline Part gastrectomy & 98 & 58 & & & 40 & 36 & \\
\hline Total gastrectomy & 116 & 77 & & & 13 & 4 & \\
\hline Pathological type & & & 0.97 & & & & 0.175 \\
\hline Adenocarcinoma & 176 & 113 & & & 44 & 38 & \\
\hline $\operatorname{mix}$ & 32 & 12 & & & 8 & 1 & \\
\hline
\end{tabular}


$P<0.05$ marked in bold font shows statistical significance.

*Adjuvant chemotherapy after surgery, no radiotherapy was administered to anyone of the patients enrolled.

\section{Results}

\section{Decreased LHPP expression correlated with poor prognosis in patients with GC}

To identify genes with prognostic value in GC, we performed transcriptome sequencing on the tumour and adjacent tissues of patients with GC and combined it with the GEO database for survival analysis to screen out the downregulated LHPP gene (Figure 1A and Supplementary Figure 1). In the TCGA database, we found that the expression of LHPP in GC was significantly lower than that in normal tissues (Figure 1B), which was consistent with our experimental results, and the expression of LHPP in GC tissues at the mRNA level (Figure 1C) and protein level (Figures 1D and 1E) were both lower than those in normal tissues. We further used IHC to evaluate the prognostic role of LHPP in GC. LHPP expression in normal tissues was significantly higher than that in gastric tumour tissues (Figures $1 \mathrm{~F}$ and $\mathrm{G}$ ).

To further evaluate the value of LHPP in clinical prognosis and the benefit of chemotherapy, survival analyses revealed that the overall survival rate of patients with low LHPP expression was significantly lower than that of patients with high LHPP expression (OS, $47.2 \%$ vs $63.0 \%, P=0.005)$ (Figure 1H). A further stratified analysis demonstrated that high expression of LHPP could significantly improve survival in patients receiving chemotherapy $(P=0.009)$, whereas patients with low LHPP expression experienced no significant impact on survival, regardless of whether they received chemotherapy $(P=0.092)$ (Figure 1I). In addition, the same results were observed in the external validation set. Survival analysis showed that patients with high LHPP expression had significantly better overall survival than those with low LHPP expression (OS, $85.0 \%$ vs. $60.4 \%, P=0.012$ ) (Figure $1 \mathrm{~J}$ ). Same as the internal set, whether patients with high LHPP expression received chemotherapy had a significant difference in their survival rate $(P=$ 0.022), while patients with low LHPP expression had no difference $(P=0.292)$ (Figure $1 \mathrm{~K})$. Collectively, these results suggest that LHPP has potential clinical value as a predictive biomarker for disease outcome in GC.

\section{LHPP suppressed the proliferation and metastasis of GC cells in vitro}

To investigate the potential role of LHPP in the invasion and metastasis of GC, we detected the expression level of LHPP in several GC cell lines (Figure 2A) and constructed HGC-27 and MGC-803 GC cell lines with stable overexpression or downregulation of LHPP (Figure 2B). We found that knockdown of LHPP expression promoted the proliferation, migration, and invasion ability of MGC-803 cells in vitro; in contrast, upregulated LHPP expression levels resulted in a significant reduction in the proliferation, 
migration, and invasion ability (Figures 2C-2E). In addition, LHPP affected drug resistance. The plate clone and IC50 of the drug resistance experiment showed that the knockdown of LHPP increased the drug resistance of GC cells in vitro, whereas overexpression of LHPP caused a decrease in drug resistance of GC cells in vitro (Figures 2F-2G), which was verified in two different cell lines (MGC-803 and MKN-28) (Supplementary Figure 2). Thus, these results demonstrated that LHPP suppressed GC tumorigenicity in vitro.

\section{LHPP suppressed the proliferation and metastasis of GC in vivo}

To further verify the biological functions of LHPP in vivo, we constructed a subcutaneous tumour model in nude mice to evaluate the effect of LHPP on the tumorigenic ability of GC cells in vivo. After the overexpression of LHPP, tumour growth was inhibited, and the growth in tumour volume was significantly decelerated compared with that in the control group. After knocking down LHPP, the results were reversed. When establishing the nude mouse subcutaneous tumour model, we divided the nude mice into two groups: one group was injected with $0.9 \% \mathrm{NaCl}$, and the other group was treated with the chemotherapy drug oxaliplatin. After 4 weeks, the cells overexpressing LHPP in the oxaliplatin group showed stronger drug sensitivity than those in the control group, whereas the cells with knocked down LHPP showed stronger drug resistance than those in the control group (Figures $3 \mathrm{~A}$ and $3 \mathrm{~B}$ ).

To determine the role of LHPP in GC metastasis in vivo, MGC-803 cells overexpressing LHPP or corresponding control cells were injected into nude mice via the tail vein. When investigating the lungs 4 weeks after injection, mice injected with LHPP-overexpressing cells exhibited significantly reduced GC lung metastasis, as shown by bioluminescence imaging (Figure 3C). The number of metastatic colonies in the control group was higher than that in the LHPP-overexpression group (Figure 3D). Histological analysis of the dissected lungs using H\&E staining confirmed that the control group had more metastatic nodules than the LHPP-overexpression group (Figure 3E). Furthermore, enhanced LHPP expression significantly inhibited peritoneal metastasis after the intraperitoneal injection of GC cells. When establishing the tumour metastasis model by intraperitoneal injection, we also divided the nude mice into two groups: one group was injected with $0.9 \% \mathrm{NaCl}$, and the other group was treated with the chemotherapy drug oxaliplatin. After 4 weeks, nude mice in the oxaliplatin group injected with cells overexpressing LHPP had a much greater reduction in the number of metastatic nodules in the abdominal cavity than those in the control group, indicating that high LHPP expression can lead to stronger drug sensitivity; however, in the nude mice injected with LHPP knockdown cells, the degree of reduction of peritoneal metastasis nodules was not significantly different from that of the control group, indicating that LHPP knockdown would result in stronger drug resistance (Figures 3F-3I). In addition, we successfully established a liver-spleen metastasis model. The results revealed that the overexpression of LHPP can inhibit the liver metastasis of GC cells, whereas knocking down LHPP can promote liver metastasis of GC cells (Figures 3J-3K and Supplementary Figure 3).

In summary, LHPP inhibited the proliferation, invasion, and metastasis of GC cells and reduced the drug resistance of GC cells in vivo. 


\section{METTL14-mediated m6A modification of LHPP mRNA in GC}

The mechanisms leading to aberrant LHPP expression remain unclear. Considering the low mRNA level of LHPP in GC, we questioned whether m6A modification regulates the mRNA stability of LHPP. The online prediction tool SRAMP (http://www.cuilab.cn/sramp) shows that there are many m6A sites with high confidence of LHPP (Figure 1A and Supplementary Figure 4A). Furthermore, using the GEPIA database to analyse the correlation between many important m6A methyltransferases and demethyltransferases and LHPP, we found that the methyltransferase METTL14 had a significantly positive correlation with LHPP (Figure 4B). Therefore, we knocked down the expression of METTL14 and found that the expression of LHPP also decreased, and vice versa (Figures 4C and 4D). In addition, TMA-based IHC revealed that the expression of METTL14 in GC was positively correlated with LHPP (Figure 4E). As shown in Figure 4F, we found that m6A modification of LHPP mRNA was positively correlated with the expression of METTL14 in GC cells. These results proved that the mRNA stability of LHPP was regulated by METTL14-mediated m6A methylation.

\section{LHPP is modified by acetylation to exert a tumour suppressor function}

To explore how LHPP molecules exert tumour biological functions, we performed an enrichment analysis of the protein modification functions of KEGG and GO (Supplementary Figure 5). The results demonstrated that LHPP was highly related to acetylation modification, and there might be acetylation sites (Figures 5A and 5B). To test whether LHPP is an acetylated protein, we analysed the acetylation of endogenous LHPP (Figure 5C) and exogenously expressed LHPP (Figure 5D) in cells treated with trichostatin A (TSA), a broad-spectrum inhibitor of the histone deacetylases (HDAC) family of deacetylases, and nicotinamide (NAM), an inhibitor of the sirtuins (SIRT) family of deacetylases. Using a specific antibody against acetylated lysine, we detected strong acetylation of both exogenous and endogenous LHPP in NAM-treated but not TSA-treated cells (Figures 5C and 5D). To clarify which phosphatase inhibitor mediates the acetylation of LHPP, we performed exogenous and endogenous acetylation of LHPP cells treated with three phosphatase inhibitors, aprotinin, pepstatin, and $\mathrm{Na}_{3} \mathrm{VO}_{4}$. Surprisingly, we found that $\mathrm{Na}_{3} \mathrm{VO}_{4}$ stimulated the acetylation of exogenous and endogenous LHPP (Figures $5 \mathrm{E}$ and $5 \mathrm{~F}$ ). These results suggest that $\mathrm{p} 62$ is an acetylated protein and that acetylation can be specifically triggered by the phosphatase inhibitor $\mathrm{Na}_{3} \mathrm{VO}_{4}$. To determine the acetyltransferase which regulates LHPP acetylation, we knocked down the acetyltransferases p300, TIP60, GCN5, and PCAF in cells overexpressing LHPP. The results revealed that knockdown of PCAF, but not the other acetyltransferase genes, largely reduced the basal acetylation level of LHPP (Figure 5G). The above results indicated that LHPP is an acetylated protein regulated by phosphatase inhibitors, and its acetyltransferase is PCAF of the Kats family. Thus, LHPP exerts a tumour suppressor effect through acetylation.

\section{LHPP suppressed aerobic glycolysis}


To explore how LHPP affects the proliferation and invasion of GC, we conducted an enrichment analysis of the Reactome pathway and the biological functions of LHPP. The results revealed that LHPP is significantly related to the Akt signalling pathway, Wnt signalling pathway, and cell energy metabolism pathways (Figure 6A). The downstream effectors of the PI3K/Akt and Wnt pathways play a central role in cancer cell metabolic reprogramming. For example, the hyperactivation of Akt promotes aerobic glycolysis and glutaminolysis, and c-Myc activates the transcription of various glycolytic and glutaminolysis genes. Therefore, we detected the expression levels of glycolysis-related proteins HIF1A, GLUT1, C-MYC, PDHK1, PKM2, ALDOL A, ENO1, LDHA, and GLS in MGC-803 cells overexpressing or with knocked down LHPP (Figures 6B and 6C). The results revealed that LHPP could inhibit glycolysis-related proteins, thereby inhibiting aerobic glycolysis in GC cells. In further immunohistochemical testing, we found that LHPP and hypoxia-inducible factor HIF1A were significantly negatively correlated (Figures 6D). In the experiment of cell oxygen consumption rate and extracellular acidification rate, overexpression/knockdown of LHPP respectively increased or decreased the cell oxygen consumption rate and decreased or increased the extracellular acidification rate (Figure 6E and Supplementary Figure 6). These results showed that LHPP could inhibit aerobic glycolysis in GC cells.

To further clarify the pathways through which LHPP affects glycolysis, we used the Human PhosphoKinase Array Kit to identify phosphorylated kinases that are closely related to LHPP. The results demonstrated that the expression levels of multiple phosphorylated protein kinases were different between the control and LHPP-overexpressing GC HGC-27 cells, among which phosphorylated GSK-3 $\beta$ and Akt levels changed most significantly (Figure 6F). ICH and western blot experiments also proved that LHPP was negatively correlated with the phosphorylation of GSK-3 $\beta$, and LHPP could also regulate the phosphorylation of $\beta$-catenin, a key molecule in the Wnt pathway (Figures $6 \mathrm{G}$ and $6 \mathrm{H}$ ). In addition, through the STRING database and endogenous co-immunoprecipitation experiments, we further proved that LHPP and GSK-3 $\beta$ interacted (Figure 61 and Supplementary Figure 6E). Further rescue experiments confirmed previous results (Supplementary Figure 7). In summary, LHPP inhibited the Wnt pathway by inhibiting the phosphorylation of GSK-3ß, thereby inhibiting the growth of GC cells.

\section{Discussion}

A key factor leading to the occurrence of aerobic glycolysis in tumour cells is that hypoxia or oncogene expression leads to the activation of hypoxia-inducible factor-1 (HIF-1) $[19,20]$. HIF-1 is a heterodimeric protein composed of a stable $b$ subunit and an unstable gene. Under positive oxygen pressure, oxygendependent hydroxyproline dioxygenase is linked to VHL. With the expression of the enzyme, the synthesized HIF-1 is subsequently degraded by VHL ubiquitination. Under hypoxic stress, HIF-1 promotes the conversion of glucose to acetone by upregulating the expression of Glut1, HK1, HK2, lactate dehydrogenase A (LDH-A), and MCT4 (monocarboxylate transporter 4), and the aerobic glycolysis of acid and lactic acid proceeds [21]. In addition, HIF-1 also activates the expression of PDK1 (pyruvate dehydrogenase kinase) through transcription while inhibiting pyruvate dehydrogenase (PDH) to convert pyruvate into acetyl-coenzyme A ( $\mathrm{Ac}-\mathrm{CoA})$, while $\mathrm{CoA}$ is normal, the raw materials which enter the tricarboxylic acid cycle and the electron transport provider in respiratory chain complexes 1 and 2 [22]. 
Therefore, by inhibiting PDH, HIF-1 restricts the progress of mitochondrial oxidative phosphorylation. In addition, HIF-1 cooperates with c-Myc to induce the expression of HK2 and PDK1, thereby further promoting the progress of aerobic glycolysis, enabling tumour cell protein synthesis, cell cycle progression and metabolic reprogramming, and finely regulating tumour cell growth and the metabolic adaptive response in a hypoxic environment [23].

The regulation of glucose metabolism genes by transcription factors is an important mechanism in tumour energy metabolism reprogramming [26]. In addition to the aforementioned HIF-1a, transcription factor FOX01, signal transducer and activator of transcription 3 (STAT3), Sp1, etc., mainly act as tumour suppressor genes in tumours [27]. Demaria et al. found that under stress conditions, tyrosine 705 is activated by phosphorylation, and transcription activates HIF-1a to promote glycolysis [28]. The specificity protein (Sp) is a member of the Sp/Kruppel-like factor family. Sp1 is overexpressed in some tumours and often interacts with the PI3K/AKT signalling pathway and other transcription factors to regulate tumour glycolysis. Kao et al. reported that endothelin-1 (ET-1) and cAMP can synergistically activate $\mathrm{Sp} 1$ to promote GLUT1 transcription, thereby regulating the glycolysis process in tumour cells [29].

The specific molecular mechanism of the high glycolysis energy metabolism phenotype of tumour cells has not yet been fully elucidated. Recent studies have shown that the regulation of aerobic oxidation is related to abnormalities in multiple signalling pathways. In addition, the opening and closing of the Wnt signalling pathway directly control the expression levels of a large number of genes related to growth and metabolism. Pate et al. found that Wnt signalling can regulate glycolysis and angiogenesis through PDK1 and interfere with colon cancer cells. Wnt signalling can reduce glycolytic metabolism and inhibit the growth of tumour cells [31]. Preliminary research by the project team also found that in GC cells, Akt can mediate the phosphorylation of GSK-3 $\beta$, which in turn affects the expression of $\beta$-catenin protein, thereby regulating GC invasion and metastasis [32-34]. Many tyrosine kinase receptors, such as epidermal growth factor receptor (EGFR) and platelet-derived growth factor receptor (PDGFR), can activate PI3K on the cell surface; subsequently, Akt is recruited to the cell and activates mTOR, which can induce HIF-1 to promote glycolysis in cells [36].

In conclusion, we found that LHPP acts as a tumour suppressor in GC by inhibiting cell proliferation, invasion, and drug resistance. We further proposed that LHPP is regulated by m6A methylation and regulates the metabolism of GC by changing the acetylation level. Thus, LHPP is a potential predictive biomarker and therapeutic target for GC (Figure 7). The study of the influence of LHPP on the metabolic Warburg effect and biological phenotype of GC and its specific mechanisms will further deepen the understanding of the metabolic adaptation, occurrence, and development of GC cells and provide new candidate targets and intervention methods for the treatment of GC.

\section{Conclusion}


LHPP is regulated by m6A methylation and regulates the metabolism of GC by changing the acetylation level. Thus, LHPP is a potential predictive biomarker and therapeutic target for GC.

\section{Abbreviations}

LHPP: Phospholysine Phosphohistidine Inorganic Pyrophosphate Phosphatase; GC: gastric cancer; RNAseq: RNA sequencing; TCGA: The Cancer Genome Atlas; qRT-PCR: quantitative reverse transcription polymerase chain reaction; GEO: Gene Expression Omnibus; IHC: immunohistochemistry; TMA: tissue microarray; GSEA: gene set enrichment analysis; STRING: Search Tool for the Retrieval of Interacting Genes; ns: no significant difference; KEGG: Kyoto Encyclopedia of Genes and Genomes; TSA: trichostatin A; NAM: nicotinamide; shRNA: short hairpin RNA; GO: Gene Ontology; METTL14: Methyltransferase Like 14; GSK3b: Glycogen Synthase Kinase 3 Beta; HIF1A: Hypoxia Inducible Factor 1 Subunit Alpha; GLUT1: Solute Carrier Family 2 Member 1; C-MYC: Proto-Oncogene, BHLH Transcription Factor; PDHK1: Pyruvate Dehydrogenase Kinase 1; PKM2: Pyruvate Kinase M2; ALDOL A: Aldolase A; ENO1: Enolase 1; LDHA: Lactate Dehydrogenase A; GLS: Glutaminase; GEPIA: Gene Expression Profiling Interactive Analysis; SYSU: Sun Yat-sen University.

\section{Declarations}

\section{Ethics approval and consent to participate}

Ethics approval was provided by the Ethics Committee of Fujian Medical University Union Hospital and the Affiliated Hospital of Qinghai University.

\section{Consent for publication}

We have received consents from individual patients who have participated in this study. The consent forms will be provided upon request.

\section{Availability of data and materials}

The datasets used and/or analyzed during the current study are available from the corresponding author on reasonable request.

\section{Competing interests}

The authors declare that they have no competing interests.

\section{Funding}

This work was supported by the National Natural Science Foundation of China (No. 81871899, $82072603,81802312)$, the Project supported by the Science Foundation of Fujian Province, China (2018J01307), the Joint Funds for the Innovation of Scientific and Technological of Fujian Province, 
China (2018Y9008) and the Health and Health Career Training Project for Young and Middle-aged Key Talents of Fujian Province, China (2020GGA033).

\section{Authors' contributions}

JX Lin, NZ Lian and YX Gao conceived the study, analyzed the data, and drafted the manuscript. CM Huang and JB Wang critically revised the manuscript for important intellectual content. QZ Qiu, HG Wang, QL Zheng, YH Yang, YB Ma, CH Zheng, P Li, JW Xie, J Lu, QY Chen, LL Cao and M Lin assisted in data collection and study design.

\section{Acknowledgements}

The authors thank the numerous patients, their families and the staff of animal facility that have participated in this study.

\section{References}

1. Siegel R, Miller K, Jemal A. Cancer statistics, 2018. CA cancer J Clin. 2018, 68(1):7-30.

2. Chen W, Zheng R, Baade PD, et al. Cancer statistics in China, 2015. CA Cancer J Clin. 2016, 66(2):115-132.

3. Hartgrink HH, Jansen EP, van Grieken NC, van de Velde CJ. Gastric cancer. Lancet. 2009, 374(9688):477-490.

5. Chae YC, Kim JH. Cancer stem cell metabolism: target for cancer therapy. BMB Rep. 2018, 51(7):319-326.

6. Mayers JR, Vander Heiden MG. Nature and nurture: what determines tumor metabolic phenotypes? Cancer Res. 2017, 77(12):3131-3134.

8. Makwana MV, Muimo R, Jackson RF. Advances in development of new tools for the study of phosphohistidine. Lab Invest. 2018, 98(3):291-303.

9. Zhang J, Gelman IH, Katsuta E, et al. Glucose drives growth factor-independent esophageal cancer proliferation via phosphohistidine-focal adhesion kinase signaling. Cell Mol Gastroenterol Hepatol. 2019, 8(1):37-60.

10. Fuhs SR, Meisenhelder J, Aslanian A, et al. Monoclonal 1- and 3-phosphohistidine antibodies: new tools to study histidine phosphorylation. Cell. 2015, 162(1):198-210.

11. Polimanti R, Wang Q, Meda SA, et al. The interplay Between risky sexual behaviors and alcohol dependence: genome-wide association and neuroimaging support for LHPP as a risk gene. Neuropsychopharmacology. 2017, 42(3):598-605. 
12. Neff CD, Abkevich V, Packer JC, et al. Evidence for HTR1A and LHPP as interacting genetic risk factors in major depression. Mol Psychiatry. 2009, 14(6):621-630.

13. Gutierrez-Camino A, Martin-Guerrero I, García-Orad A. Genetic susceptibility in childhood acute lymphoblastic leukemia. Med Oncol. 2017, 34(10):179.

14. Shiga Y, Akiyama M, Nishiguchi KM, et al. Genome-wide association study identifies seven novel susceptibility loci for primary open-angle glaucoma. Hum Mol Genet. 2018, 27(8):1486-1496.

15. Hindupur SK, Colombi M, Fuhs SR, et al. The protein histidine phosphatase LHPP is a tumour suppressor. Nature. 2018, 555(7698):678-682.

16. Chen $\mathrm{M}$, Huang J. The expanded role of fatty acid metabolism in cancer: new aspects and targets. Precis Clin Med. 2019, 2(3):183-191.

17. Koppenol WH, Bounds PL, Dang CV. Otto Warburg's contributions to current concepts of cancer metabolism. Nat Rev Cancer. 2011, 11(5):325-337.

18. Czernin J, Phelps ME. Positron emission tomography scanning: current and future applications. Annu Rev Med. 2002, 53:89-112.

19. Kelloff GJ, Hoffman JM, Johnson B, et al. Progress and promise of FDG-PET imaging for cancer patient management and oncologic drug development. Clin Cancer Res. 2005, 11(8):2785-2808.

20. Zhang T, Suo C, Zheng C, et al. Hypoxia and metabolism in metastasis. Adv Exp Med Biol. 2019, 1136:87-95.

21. Koyasu S, Kobayashi M, Goto Y, et al. Regulatory mechanisms of hypoxia-inducible factor 1 activity: two decades of knowledge. Cancer Sci. 2018, 109(3):560-571.

22. Wohlrab C, Kuiper C, Vissers MC, et al. Ascorbate modulates the hypoxic pathway by increasing intracellular activity of the HIF hydroxylases in renal cell carcinoma cells. Hypoxia. 2019, 7:17-31.

23. Zhao H, Jiang H, Li Z, et al. 2-Methoxyestradiol enhances radiosensitivity in radioresistant melanoma MDA-MB-435R cells by regulating glycolysis via HIF-1a/PDK1 axis. Int J Oncol. 2017, 50(5):1531-1540.

24. Podar K, Anderson KC. A therapeutic role for targeting c-Myc/Hif-1-dependent signaling pathways. Cell Cycle. 2010, 9(9):1722-1788.

26. Rodríguez-Enríquez S, Marín-Hernández Á, Gallardo-Pérez JC, et al. Transcriptional regulation of energy metabolism in cancer cells. Cells. 2019, 8(10):1225.

27. Haug K, Cochrane K, Nainala VC, et al. MetaboLights: a resource evolving in response to the needs of its scientific community. Nucleic Acids Res. 2020, 48:440-444. 
28. He L, Gomes AP, Wang X, et al. mTORC1 promotes metabolic reprogramming by the suppression of GSK3-dependent Foxk1 phosphorylation. Mol Cell. 2018, 70:949-960.e4.

29. Kao YS, Fong JC. A novel cross-talk between endothelin-1 and cyclic AMP signaling pathways in the regulation of GLUT1 transcription in 3T3-L1 adipocytes. Cell Signal. 2011, 23(5):901-910.

30. Jing $\mathrm{H}, \mathrm{Hu} \mathrm{J}, \mathrm{He} \mathrm{B}$, et al. A SIRT2-selective inhibitor promotes c-Myc oncoprotein degradation and exhibits broad anticancer activity. Cancer Cell, 2016, 29(3):297-310.

31. Pate KT, Stringari C, Sprowl-Tanio S, et al. Wnt signaling directs a metabolic program of glycolysis and angiogenesis in colon cancer. EMBO J. 2014, 33(13):1454-1473.

35. Courtnay R, Ngo DC, Malik N, et al. Cancer metabolism and the Warburg effect: the role of HIF-1 and PI3K. Mol Biol Rep. 2015, 42(4):841-851.

36. Shimura $\mathrm{T}$, Sasatani $\mathrm{M}$, Kawai $\mathrm{H}$, et al. Radiation-induced myofibroblasts promote tumor growth via mitochondrial ROS-activated TGF $\beta$ signaling. Mol Cancer Res. 2018, 16(11):1676-1686.

\section{Figures}


Figure 1
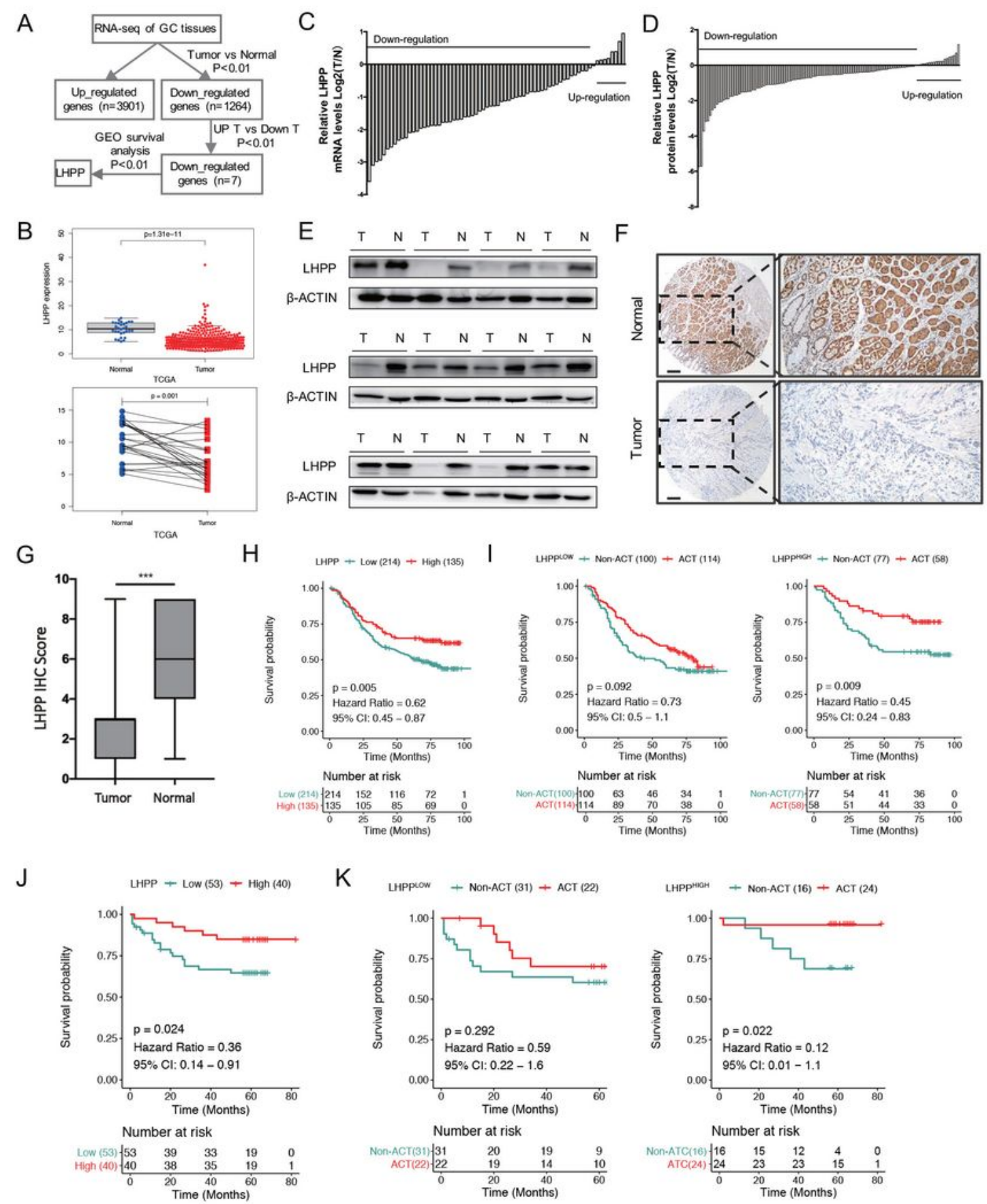

\section{Figure 1}

Expression and prognostic value of LHPP in GC. (A) Flowchart of the screening process of candidate genes. (B) Difference in LHPP protein expression between gastric tumours and adjacent normal gastric tissues in the TCGA database. (C) mRNA levels of LHPP in gastric tumours and adjacent normal tissues measured by qRT-PCR. (D) LHPP protein levels in gastric tumours and adjacent normal tissues measured by western blot. (E) Representative images of LHPP protein levels in gastric tumours and adjacent normal 
tissues. (F) Expression of LHPP in 123 paraffin-embedded specimens from the internal cohort was determined by TMA-based IHC staining. Scale bars $=200 \mu \mathrm{m}$. (G) LHPP IHC score of gastric tumours and adjacent normal tissues in Figure 1F. $(\mathrm{H})$ Kaplan-Meier analyses of the correlations between LHPP expression and overall survival in the internal cohort. (I) Kaplan-Meier analyses of the correlations between chemotherapy and overall survival in the internal cohort stratified by LHPP expression. $(J)$ Kaplan-Meier analyses of the correlations between LHPP expression and overall survival in the external cohort. (K) Kaplan-Meier analyses of the correlations between chemotherapy and overall survival in the external cohort stratified by LHPP expression. ${ }^{* *} \mathrm{P}<0.001 \mathrm{GC}$, gastric cancer; TGCA, The Cancer Genome Atlas; qRT-PCR, quantitative reverse transcription-polymerase chain reaction; TMA, tissue microarray; IHC, immunohistochemistry 
Figure 2

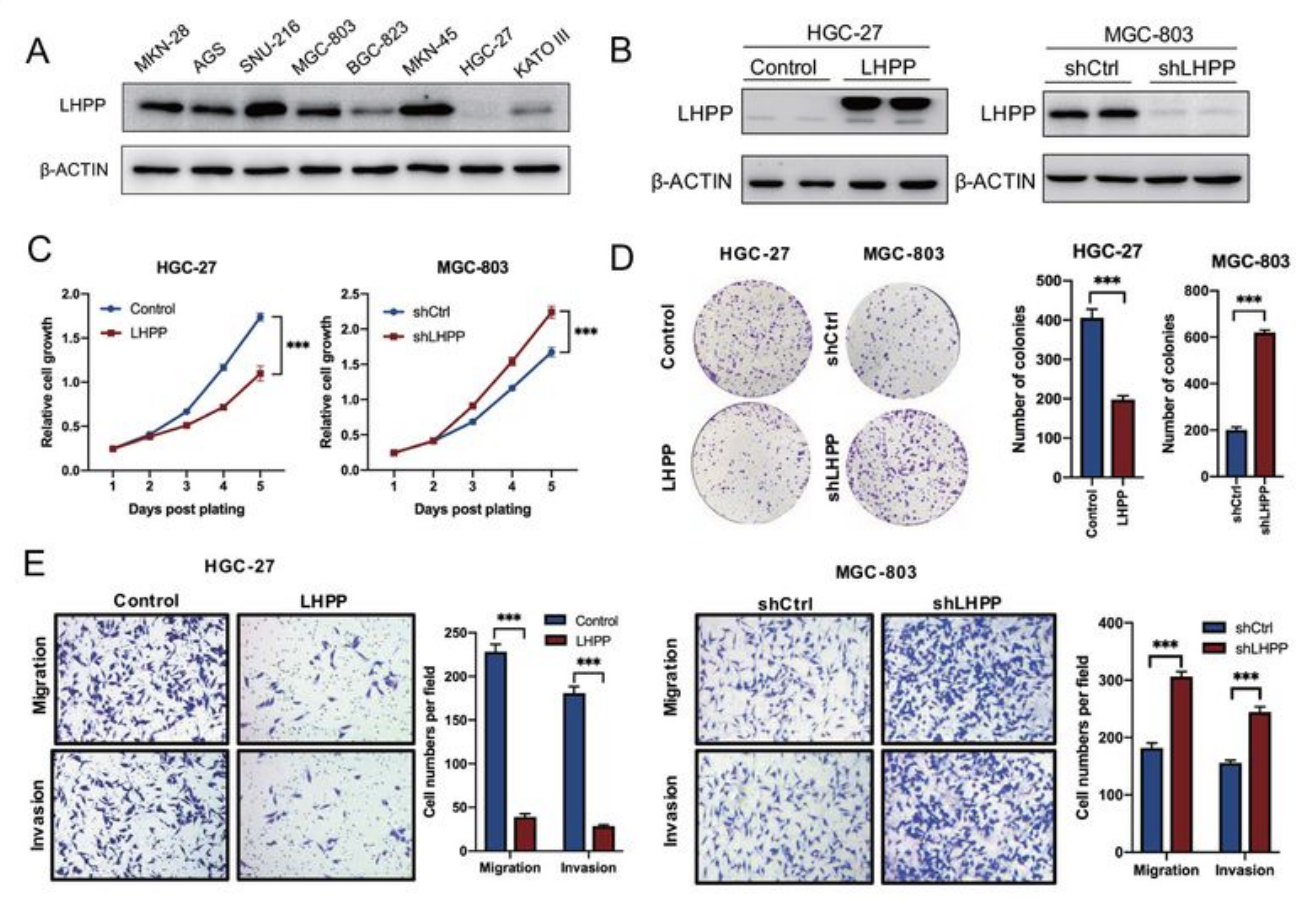

F
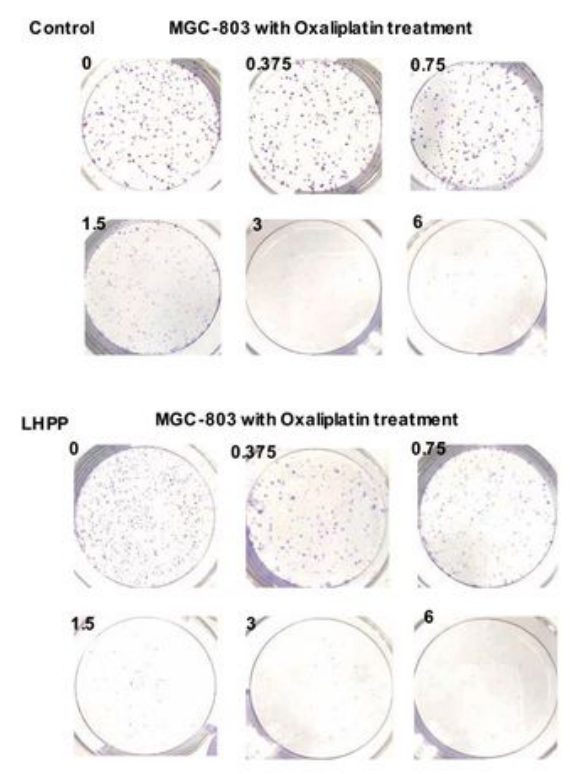

G
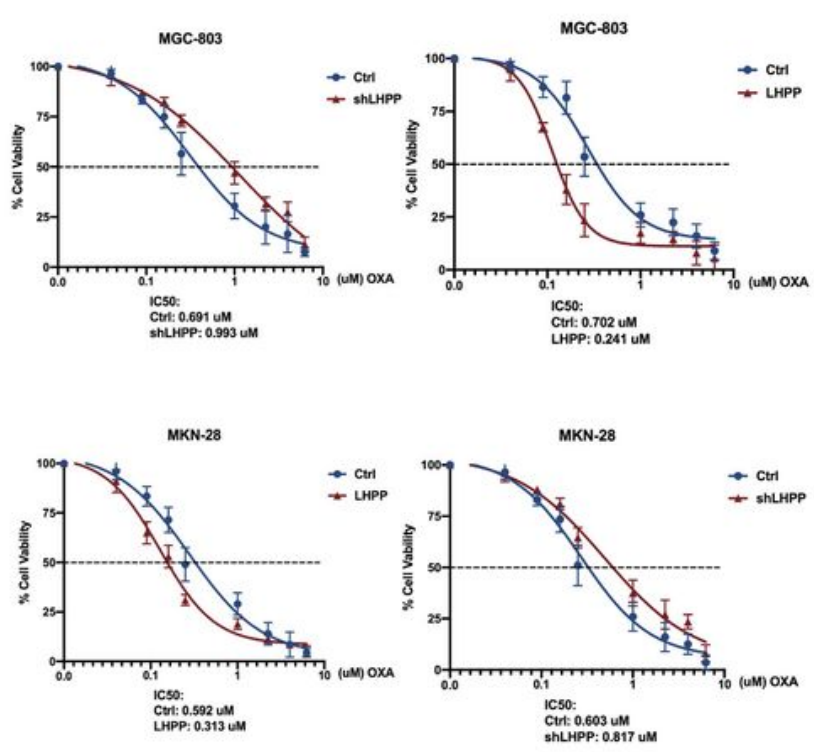

\section{Figure 2}

LHPP inhibits GC proliferation and metastasis in vitro. (A) Basic protein expression of LHPP in GC cell lines (MKN-28, AGS, SNU-216, MGC-803, BGC, MKN-45, HGC-27, and KATO III) was detected by western blotting. (B) HGC-27 cells with stable LHPP overexpression or MGC-803 cells with LHPP knockdown were created. The changes in LHPP expression were confirmed using western blotting. (C, D) The proliferative ability of stably transfected HGC-27 or MGC-803 cells was investigated via CCK-8 assays and colony 
formation. (E) Transwell assays with stably transfected HGC-27 and MGC-803 cells were performed. Representative images and quantification of the results are presented. $(F, G)$ The drug resistance of stably transfected HGC-27 or MGC-803 cells was investigated via IC50 assays and colony formation. $\star \star \star P<0.001 \mathrm{GC}$, gastric cancer

Figure 3

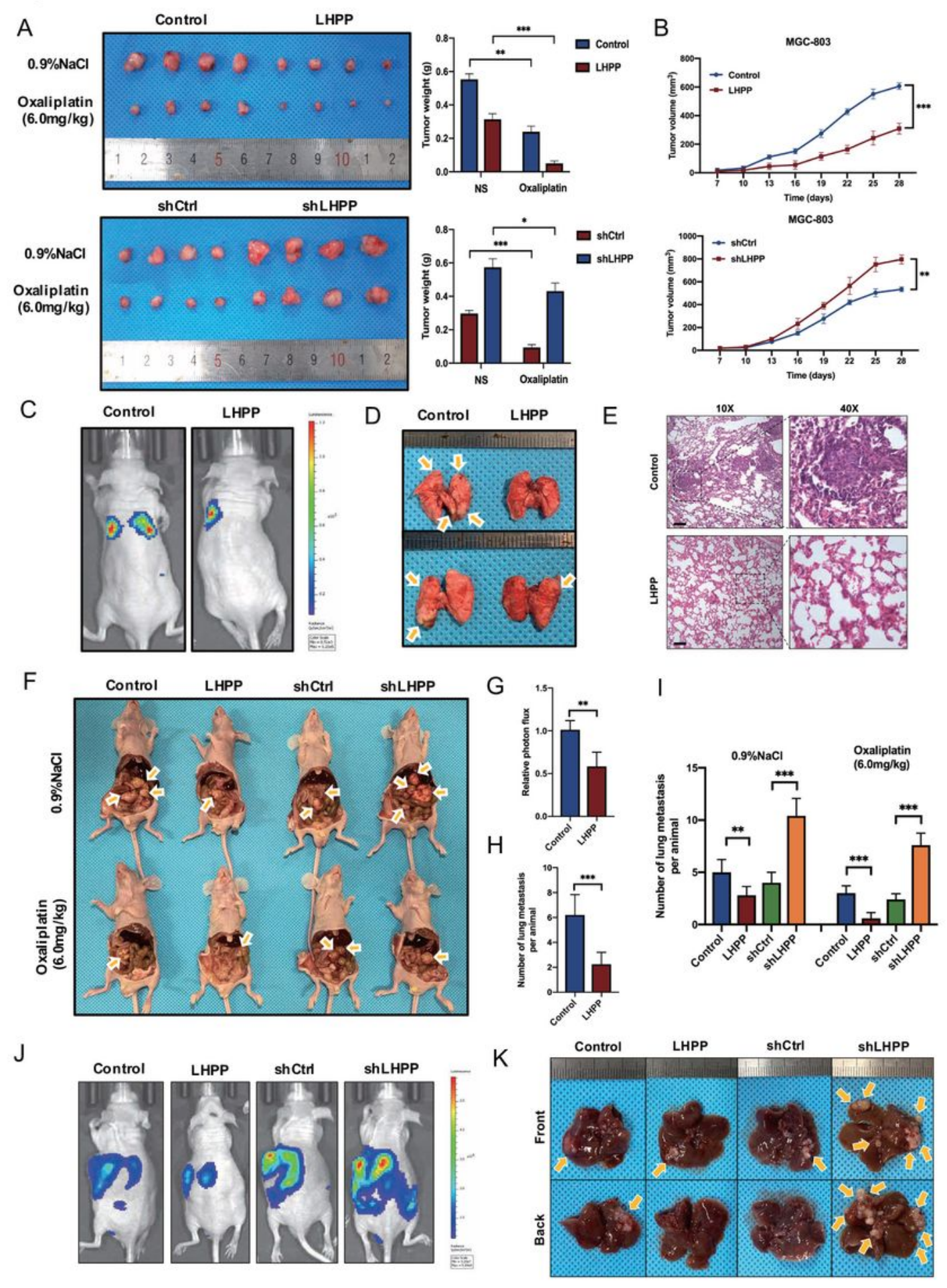

Figure 3 
LHPP inhibits GC proliferation and metastasis in vivo. (A) Overexpression of LHPP inhibited GC growth in a subcutaneous xenograft model. LHPP knockdown promoted GC growth in a subcutaneous xenograft model. The size of the tumours was measured at the indicated time points. Tumours were extracted and weighed after mice were sacrificed. (B) Representative bioluminescence images of mice at 4 weeks after tail vein injection of LHPP-overexpressing MGC-803 cells or control cells and quantification of the images. (D, E) Representative images of lung metastasis and hematoxylin-and-eosin staining. Metastatic nodules were counted with or without a microscope and recorded. Overexpression of LHPP in MGC-803 cells significantly reduced the number of metastatic lesions in the lungs. Scale bars $=200 \mu \mathrm{m} .(\mathrm{F}-\mathrm{I})$ Stably transfected MGC-803 cells were injected intraperitoneally, and the number of metastases in the colonic wall was recorded 4 weeks later. Peritoneal metastases were examined and recorded. $(\mathrm{J})$ Representative bioluminescence images of mice at 4 weeks after spleen injection of LHPPoverexpressing or LHPP-knockdown MGC-803 cells or control cells and quantification of the images. (K) Representative images of liver metastasis. Metastatic nodules were counted with or without a microscope and recorded. Overexpression of LHPP in MGC-803 cells significantly reduced the number of metastatic lesions in the livers. Knockdown of LHPP in MGC-803 cells significantly increased the number of metastatic lesions in the livers. ${ }^{*} P<0.05,{ }^{*} P<0.01,{ }^{*} * P<0.001 \mathrm{GC}$, gastric cancer 
Figure 4

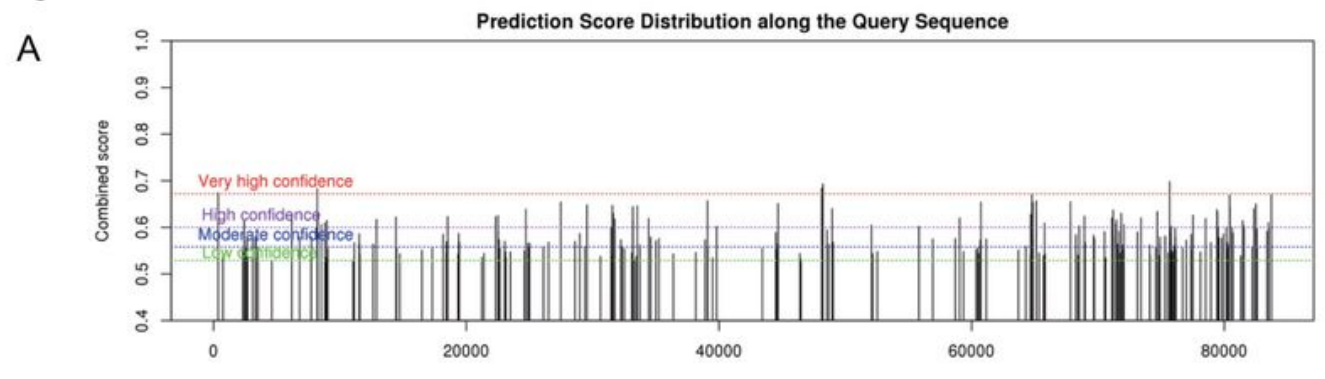

B
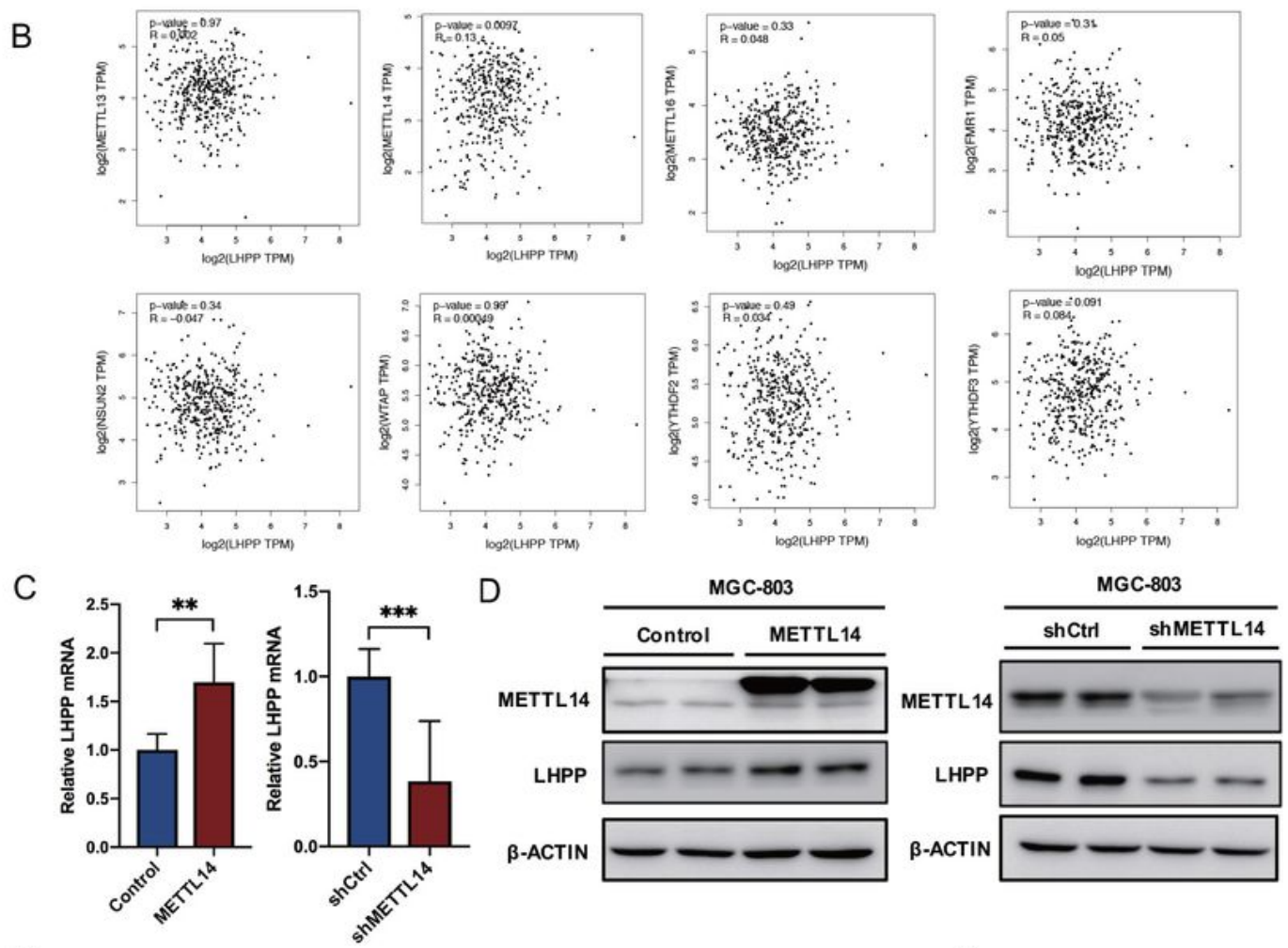

E
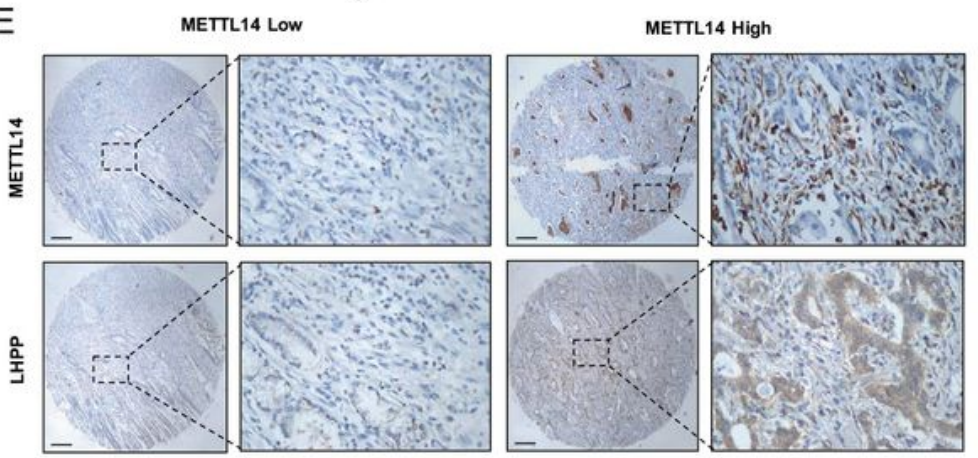

F

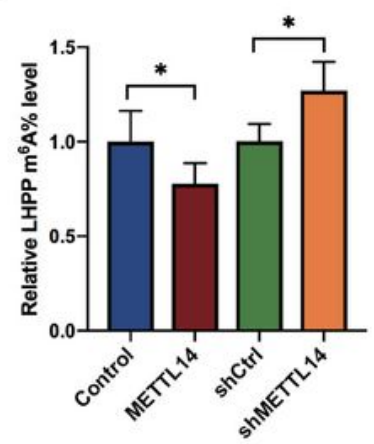

\section{Figure 4}

METTL14-mediated m6A modification represses LHPP expression in GC. (A) The potential m6A sites were predicted by SRAMP. (B) Correlation of LHPP and METTL13, METTL14, METTL16, FMR1, NSUN2, WTAP, YTHDF2, YTHDF3 according to the GEPIA database. (C-D) qRT-PCR and western blot assays revealed the mRNA and protein expression, respectively, of LHPP in GC cells with knockdown or overexpression of METTL14. (E) IHC staining of LHPP and METTL14 in TMAs. Scale bars = $200 \mu \mathrm{m}$. (F) 
m6A immunoprecipitation and qRT-PCR assays showed the relative percentage of LHPP mRNA with

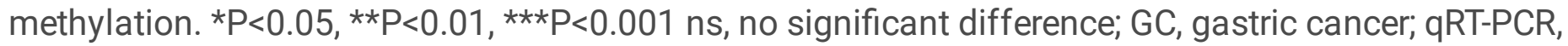
quantitative reverse transcription polymerase chain reaction; IHC, immunohistochemical; TMS, tissue microarrays; GEPIA, Gene Expression Profiling Interactive Analysis.

Figure 5

A

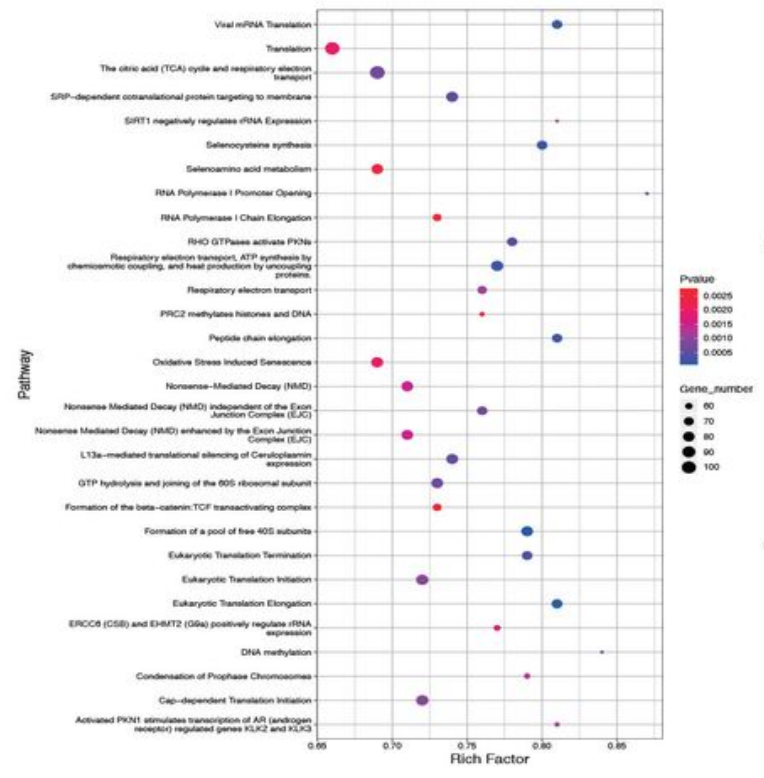

C

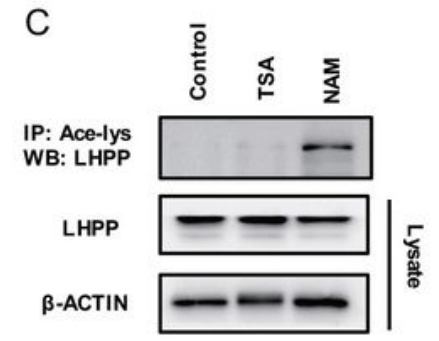

$\mathrm{D}$
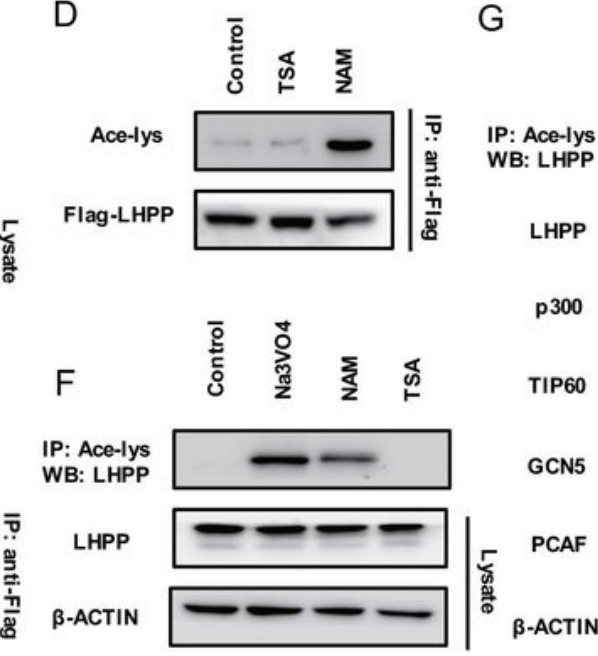

G

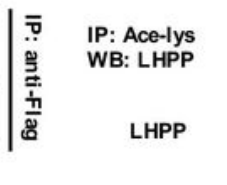

B
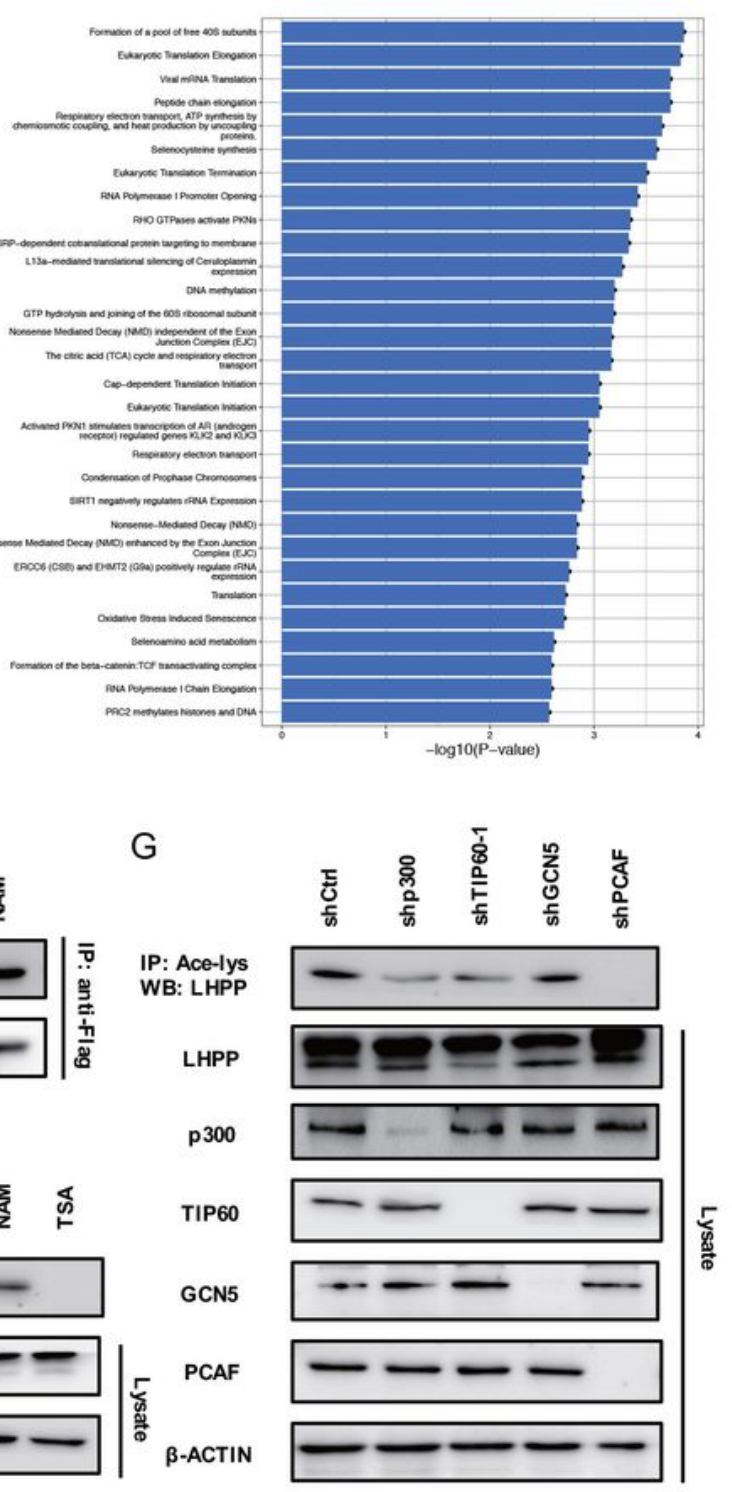

Figure 5 
LHPP modified by acetylation to exert a tumour suppressor function. (A-B) Enrichment analysis of LHPP protein modification functions of KEGG and GO. (C) Acetylation of exogenous Flag-LHPP in MGC-803 cells treated with deacetylase inhibitors, TSA or NAM. Flag-LHPP was immunoprecipitated with anti-Flag, and the precipitates were analysed using an anti-acetyl-lys antibody (Ace-lys). (D) Acetylation of endogenous LHPP in MGC-803 cells treated with TSA or NAM. LHPP acetylation was analysed using immunoprecipitation with an anti-acetyl-lys antibody followed by western blotting for LHPP. (E) Acetylation of Flag-LHPP in MGC-803 cells treated with phosphatase inhibitors aprotinin, pepstatin, and Na3V04. Flag-LHPP was immunoprecipitated with anti-Flag and analysed by western blot using antiphospho-serine. (F) Na3VO4 induced the acetylation of endogenous LHPP in MGC-803 cells on western blot. (G) Acetylation of LHPP in MGC-803 cells infected with lentivirus expressing each of the indicated acetyltransferase shRNAs. KEGG, Kyoto Encyclopedia of Genes and Genomes; GO, Gene Ontology; TSA, trichostatin A; NAM, nicotinamide; shRNA, short hairpin RNA 
Figure 6

A
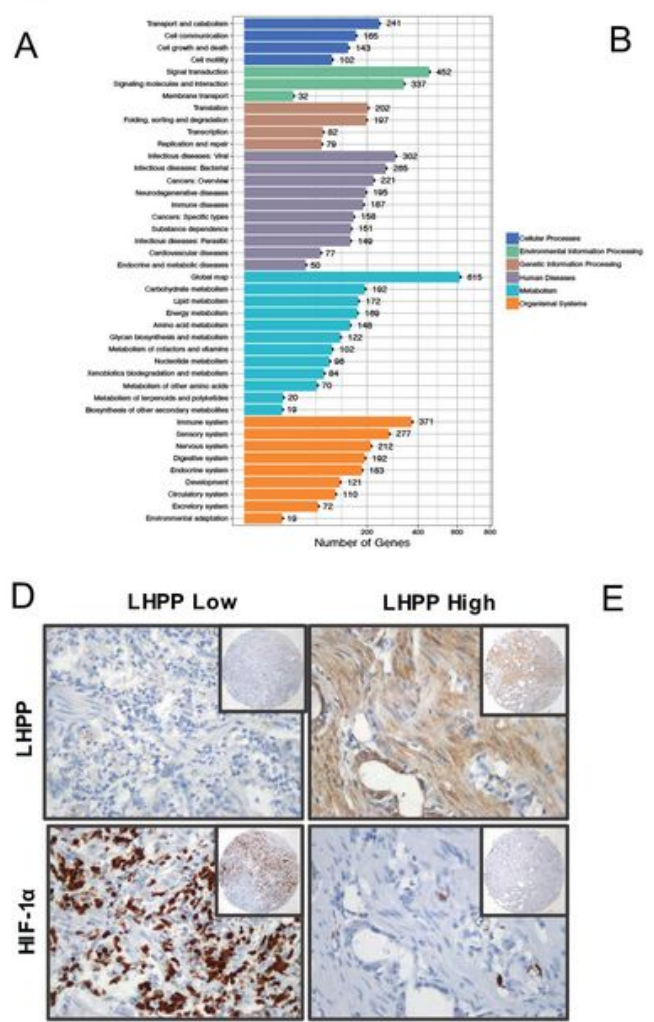

E
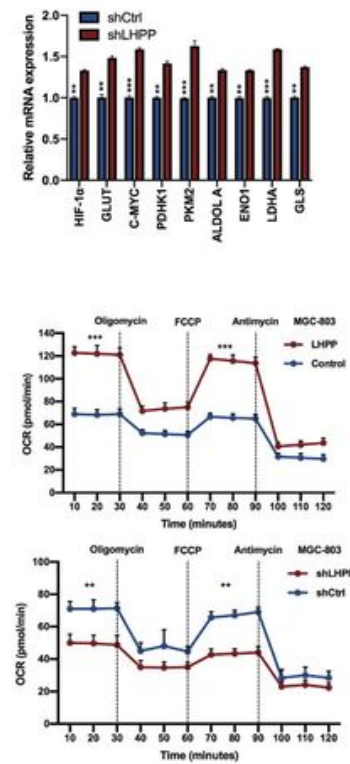

$C_{\text {MGC-803 }}$ 竞
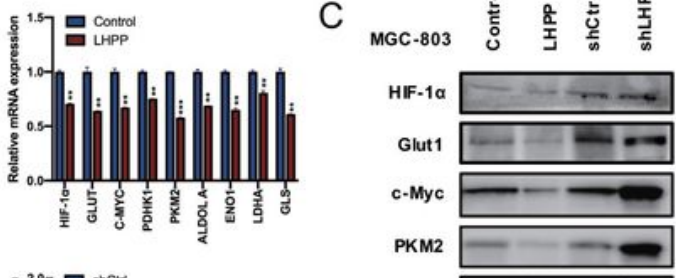

Aldolase A $\longrightarrow$

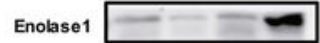

GLS1

B-ACTIN $-\longrightarrow$
F
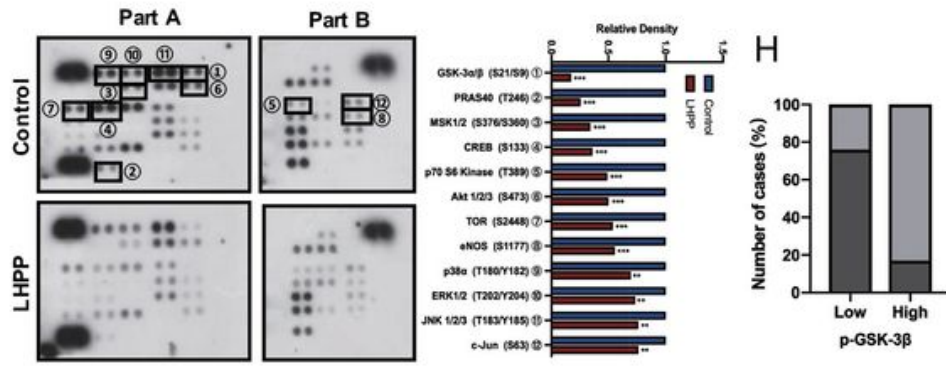

G

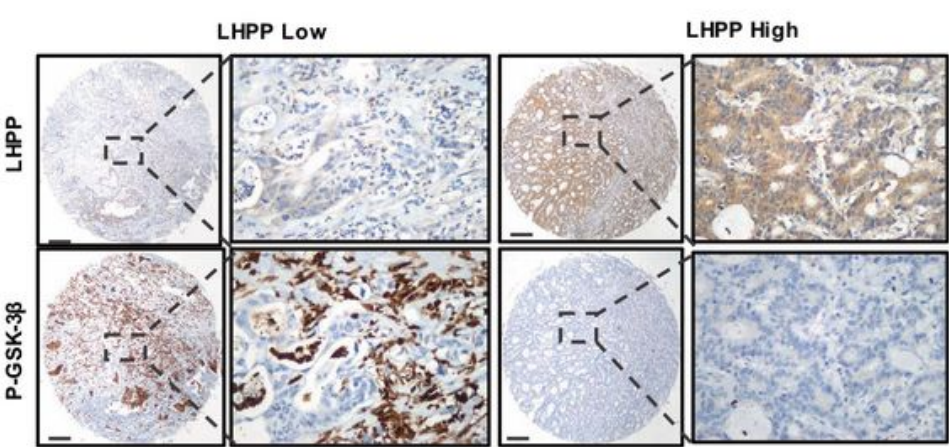

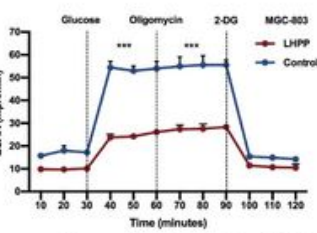

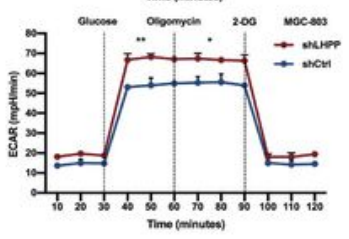

।
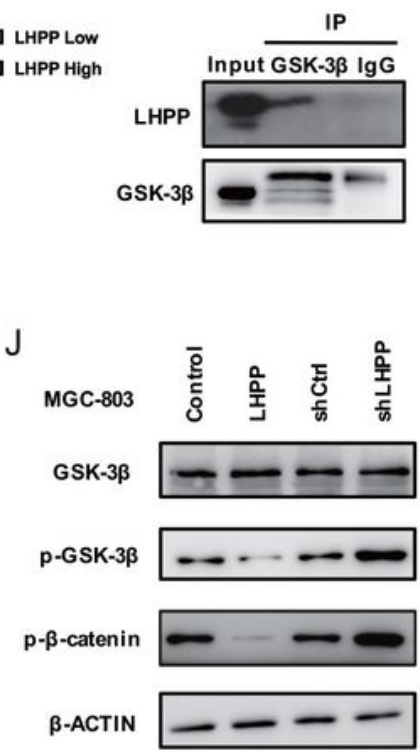

Figure 6

LHPP suppresses aerobic glycolysis. (A) Enrichment analysis of LHPP protein functions of KEGG and GO. (B) Relative mRNA expression levels of glycolytic genes and glutamine transporters in LHPPoverexpressing or LHPP-knockdown MGC-803 cells and control cells by qPCR. (C) Protein expression levels of glycolytic genes and glutamine transporters in LHPP-overexpressing or LHPP-knockdown MGC803 cells and control cells on western blot. (D) IHC staining of LHPP and HIF1A in TMAs and their 
correlation. Scale bars $=200 \mu \mathrm{m}$. (E) Oxygen consumption rate and extracellular acidification rate of LHPP-overexpressing or LHPP-knockdown MGC-803 cells and control cells measured using the Seahorse Bioscience XF96 analyser. (F) Human phosphokinase microarray assay analysis of the conditioned medium from stably transfected HGC-27 cells. A summary of the relative signal intensities of the indicated proteins is shown. (G-H) IHC staining of LHPP and p-GSK3b in TMAs and their correlation. Scale bars $=200 \mu \mathrm{m}$. (I) Combined LHPP and GSK3b by co-immunoprecipitation. (J) Protein expression levels of the Wnt pathway in LHPP-overexpressing or LHPP-knockdown MGC-803 cells and control cells on western-blot. ${ }^{*} P<0.05,{ }^{*} P<0.01,{ }^{*} * \mathrm{P}<0.001 \mathrm{KEGG}$, Kyoto Encyclopedia of Genes and Genomes; $\mathrm{GO}$, Gene Ontology; qPCR, quantitative polymerase chain reaction; IHC, immunohistochemical; TMA, tissue microarray;

Figure 7

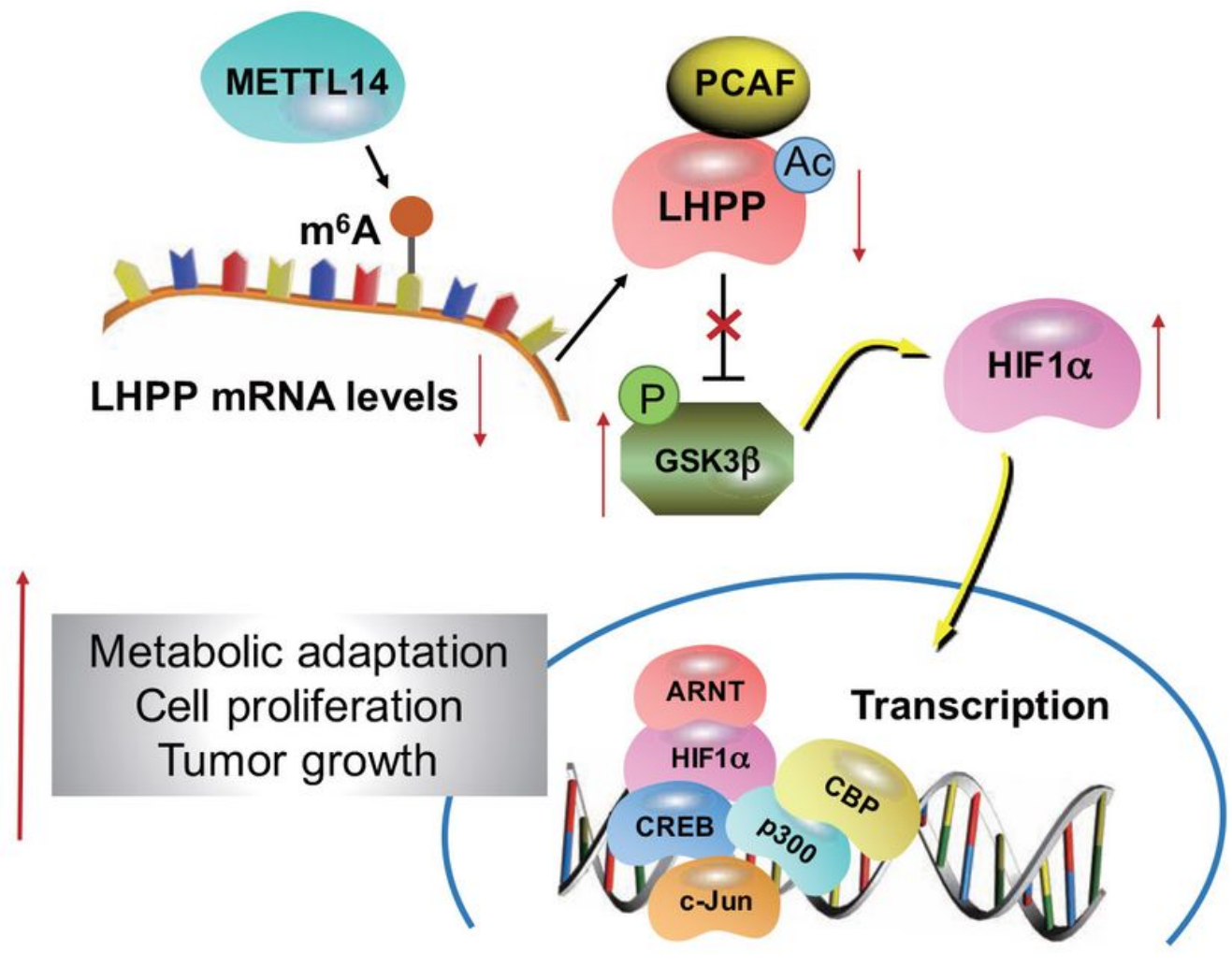

Figure 7

Proposed mechanism scheme of LHPP in GC. The m6A modification of LHPP mRNA by METTL14 represses its expression; LHPP inhibits the phosphorylation of GSK3b through acetylation, and mediates HIF1A to inhibit glycolysis, proliferation, invasion and metastasis of gastric cancer cells.

\section{Supplementary Files}


This is a list of supplementary files associated with this preprint. Click to download.

- LHPPsupplementary0517.pdf 